\title{
Highly uniform, strongly correlated fluorinated lipid nanodomains embedded in biological membrane models
}

Cite as: Appl. Phys. Lett. 93, 213901 (2008); https://doi.org/10.1063/1.3028088

Submitted: 12 September 2008 • Accepted: 25 October 2008 • Published Online: 25 November 2008

Jochen Oelke, Andreea Pasc, Achim Wixforth, et al.

\section{ARTICLES YOU MAY BE INTERESTED IN}

Native supported membranes: Creation of two-dimensional cell membranes on polymer supports (Review)

Biointerphases 3, FA12 (2008); https://doi.org/10.1116/1.2905233

First order melting transitions of highly ordered dipalmitoyl phosphatidylcholine gel phase membranes in molecular dynamics simulations with atomistic detail

The Journal of Chemical Physics 135, 055105 (2011); https://doi.org/10.1063/1.3615937

Surface acoustic wave mediated exciton dissociation in a $\mathrm{ZnCdSe} / \mathrm{LiNbO}_{3}$ hybrid

Applied Physics Letters 94, 193505 (2009); https://doi.org/10.1063/1.3136862

四 QBLOX

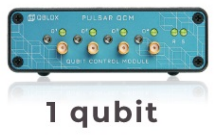

Shorten Setup Time Auto-Calibration More Qubits

Fully-integrated Quantum Control Stacks Ultrastable DC to $18.5 \mathrm{GHz}$ Synchronized $<<1$ ns Ultralow noise

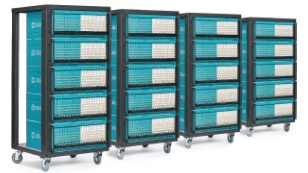

100s qubits

visit our website > 


\title{
Highly uniform, strongly correlated fluorinated lipid nanodomains embedded in biological membrane models
}

\author{
Jochen Oelke, ${ }^{1,2}$ Andreea Pasc, ${ }^{3, a)}$ Achim Wixforth, ${ }^{2}$ Oleg Konovalov, ${ }^{4}$ and \\ Motomu Tanaka ${ }^{1,3, b)}$ \\ ${ }^{1}$ Biophysical Chemistry II and BIOQUANT, University of Heidelberg, D69120 Heidelberg, Germany \\ ${ }^{2}$ Department of Physics EP1, University of Augsburg, D86159 Augsburg, Germany \\ ${ }^{3}$ Department of Physics E22, Technical University Munich, D85748 Garching, Germany \\ ${ }^{4}$ European Synchrotron Radiation Facility (ESRF), 38053 Grenoble Cedex 9, France
}

(Received 12 September 2008; accepted 25 October 2008; published online 25 November 2008)

\begin{abstract}
Synthetic, fluorinated lipids incorporated into phospholipid membranes form highly uniform self-assembled lipid nanodomains. The size of nanodomains beyond optical resolution can quantitatively be determined using grazing-incidence $\mathrm{x}$-ray diffraction. The long-range interaction can reach up to the distance that is eight times larger than the domain size, which can be interpreted within the theoretical framework of two-dimensional colloidal crystallization. Coupling of bioactive molecules to lipid head groups would allow for the creation of highly uniform and strongly correlated functional nanodomains, which can be used to study the influence of molecular confinement on the cooperative interactions in biology. () 2008 American Institute of Physics. [DOI: $10.1063 / 1.3028088]$
\end{abstract}

Biological membranes are key components of all living systems, acting as the boundary between interior and exterior of living cells. They mainly consist of a lipid bilayer membrane that possesses a fluid character. Consequently, selfassemblies of lipids and membrane proteins need not always mix uniformly but are able to form domains. The domains can be relatively static (e.g., lipid rafts and protein clusters), ${ }^{1}$ but they can also be dynamic like the accumulation of ligand-receptor complexes mediating adhesion. Both types of functional domains allow for multivalent interactions that enhance their cooperative functions. ${ }^{2}$ A commonly taken strategy to model static lipid rafts is to take similar lipid mixtures to natural ones, such as complex mixtures of sphingolipid, cholesterol, and phospholipids. ${ }^{3-5}$ However, almost all domains formed in complex lipid mixtures are found to be either highly polydispersive, or coalesce into large domains, whose diameters are often over several micrometers.

Here, we demonstrate that custom synthesized lipids with fluorocarbon chains can form highly uniform submicrometer domains, which can potentially be used as an alternative to the existing raft models. The synthetic fluorinated lipid (FL17) and the supported membrane incorporating domains of fluorinated lipids used in this study are schematically depicted in Fig. 1(a). A phospholipid monolayer incorporating FL17 is transferred from the air-water interface by horizontal dipping of a glass slide functionalized with a hydrophobic silane monolayer. ${ }^{6}$ Figure $1(\mathrm{~b})$ represents a fluorescence image of the monolayer with FL17 $=33 \mathrm{~mol} \%$, taken on an inverted microscope (Zeiss Axiovert 200) with an oil immersion objective $(100 \times, N A=1.3)$. Since the fluorocarbon chains of FL17 molecules adopt a solid, helical conformation even at a low surface pressure (see supplemental information for more details ${ }^{7}$ ), any fluorescent lipid tracers are all excluded from the fluorinated domains into fluid

\footnotetext{
${ }^{a)}$ Present address: UMR 7565, Faculté des Sciences et Techniques, Université H. Poincaré, 54506 Vandoeuvre-lès-Nancy, France.

b) Author to whom correspondence should be addressed. Electronic mail: tanaka@uni-heidelberg.de. Tel.: +49-6221544916.
}

lipid matrix. FL17 domains below the threshold intensity can be identified as dark/red spots using the CELLENGER software (Definiens AG, Munich, Germany). Since fluorescence images taken at the air-water interface and on solid-liquid interface are identical, we use the transferred monolayers for the further image analysis. 2D Fourier transform of more than 50 images [Fig. 1(c)] and subsequent radial integration of the circular halo (inset) suggest the characteristic lengths
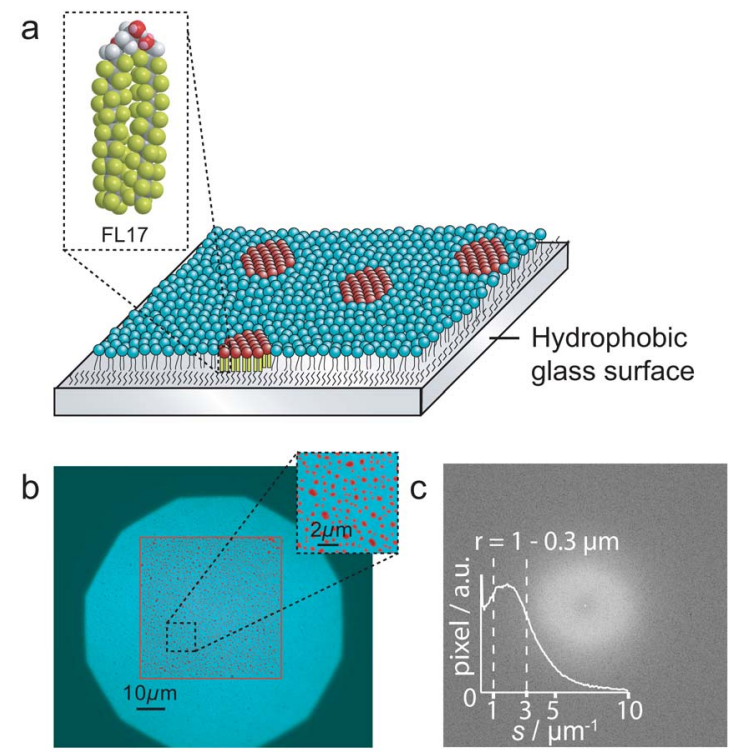

FIG. 1. (Color online) (a) Schematic illustration of a supported lipid monolayer displaying domains of FL17. Molecular structure of FL17 (space filling model) is presented as an inset. (b) Fluorescence image of a supported phospholipid monolayer with $33 \mathrm{~mol} \%$ FL17 and $0.1 \mathrm{~mol} \%$ TR-DHPE. The region of interest $\left(50 \times 50 \mu \mathrm{m}^{2}\right.$, indicated by the red/dark square) is sampled from each image, and $>50$ images are subjected to the image analysis. The domains of FL17 (highlighted in dark/red) are identified from the surrounding phospholipids matrix (blue/background) by the contrast in the pixel values. The zoom-up image (inset) suggests the formation of submicrometer domains. (c) 2D Fourier transformation of $>50$ images would reveal a broad halo around the center. Radial integration of the circular halo (inset) the characteristic lengths $0.3-1 \mu \mathrm{m}$. 
a

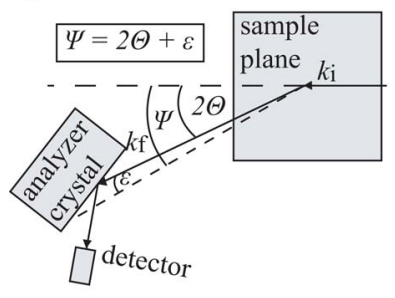

$\mathrm{b}$

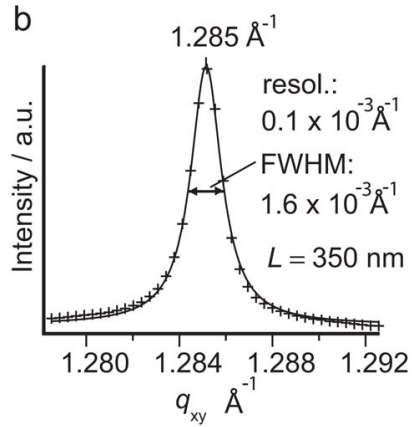

FIG. 2. (Color online) (a) Schematic view of the analyzer crystal setup used for GID measurements. (b) The intensity of the diffracted beam from the monolayer with $33 \mathrm{~mol} \%$ FL17, integrated along $q_{\mathrm{z}}$. The complete reciprocal space map is available online [supplemental Information (Ref. 7)]. The characteristic distance between fluorocarbon chains $(d=5.65 \AA)$ and the size of crystallites $(L=350 \mathrm{~nm})$ can be calculated, respectively.

of about 0.3 and $1 \mu \mathrm{m}$, which seems to coincide with the size of domains and their mean distance, respectively.

However, it should be noted that the determination of the size of submicron objects from a pixel analysis might be erroneous. Therefore, we measure grazing-incidence $\mathrm{x}$-ray diffraction (GID) of the same monolayer to determine the size of submicrometer domains in a quantitative manner. These experiments are carried out at the air-water interface at several different locations so that one can minimize artifacts from radiation damage or sample inhomogeneity. Here, we use a $\mathrm{Si}$ (111) analyzer crystal optics [Fig. 2(a)] instead of a commonly used Soller collimator to gain the higher angular resolution $\left(\Delta q=10^{-4} \AA^{-1}\right)$. The reconstructed reciprocal space map (supporting information $\mathrm{S} 1)^{7}$ clearly exhibits one very sharp in-plane peak with the intensity maximum at $q_{z}$ $=0$, denoting that fluorocarbon chains reside on a hexagonal lattice without tilting to the neighboring chains. The integrated intensity along $q_{\mathrm{z}}$ is presented in Fig. 2(b). From the peak position $\left(q_{\mathrm{xy}}=1.285 \AA^{-1}\right)$, a short-range correlation length of $4.89 \AA$ can be calculated. The corresponding distance between fluorocarbon chains of $d=5.65 \AA$ agrees well with the value reported from the crystallography of ordered fluorocarbon chains. ${ }^{8}$ The fact that fluorocarbon chains are arranged in a hexagonal lattice with almost no strain gives additional evidence that the FL17 domains remain in the plane of the membrane, and they do not form hemispherical micelles as has been reported earlier for single chain surfactants with partial fluorination. ${ }^{9}$ Moreover, taking the full width at the half maximum of the peak, (FWHM=1.6 $\times 10^{-3} \AA^{-1}$ ), we can calculate the coherent length, i.e., the size of a crystallite, of $L=350 \mathrm{~nm}$ according to the Scherrer equation $^{10}, \quad L=0.9 \times 2 \pi / \sqrt{\mathrm{FWHM}^{2}-\Delta q_{x y}^{2}}$. The obtained value agrees well with the shorter characteristic length suggested by the image analysis $[\sim 0.3 \mu \mathrm{m}$, see Fig. 1(c)], and thus we conclude that each FL17 domain consists of one single crystallite. Although the in-plane $\mathrm{x}$-ray diffraction technique with an analyzer optics has been used to elucidate the viscoelasticity of fatty acid monolayers in a solid state, ${ }^{11}$ this is one of the first demonstrations employing the analyzer crystal optics to resolve the submicrometer organic crystallites diluted in two-dimensional (2D) fluid matrices, owing to the very high in-plane ordering and high electron density of fluorocarbon chains.

The fact that self-assembled FL17 domains retain their size at various molar fractions $(5-33 \mathrm{~mol} \%)$ enables us to

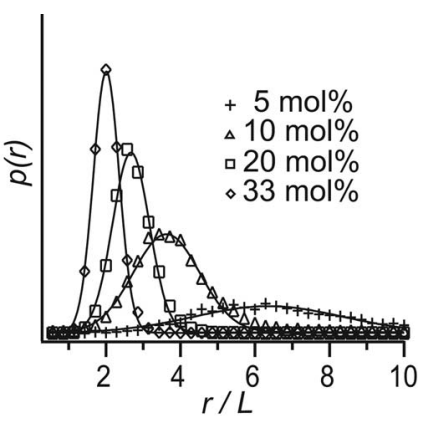

FIG. 3. Distribution function of the distance to the nearest neighbor $p(r)$ plotted vs $r / L$. The black line represents a Gaussian fit for each molar fraction. Though the decrease in FL17 leads to an increase in the peak position and width, a distinct peak can be observed even at FL17 $=5 \mathrm{~mol} \%$.

describe and regard them as correlated 2D colloidal particles. ${ }^{12}$ To estimate the strength of the lateral correlation, we extract the distributions of the distance $r$ between the nearest neighbors (distance between the centers of mass defined by the pixel analysis) for more than 2000 domains. The distribution function $p(r)$ plotted versus $r / L$ are presented in Fig. 3, where $r$ is normalized to the domain size $L$ $=350 \mathrm{~nm}$. In this plot, the peak positions correspond to the mean interdomain distance $\langle r\rangle$ at each molar concentration. As shown in the figure, $\langle r\rangle$ has a very sharp peak for the membrane with $33 \mathrm{~mol} \%$ of FL17. This finding suggests that each domain is confined at a finite distance from the neighbors but does not merge with others to minimize the boundary length. On the other hand, the peak position as well as the peak width systematically increases according to the decrease in the molar fraction of FL17. Nevertheless, it is notable that a distinct peak can be found even for the membrane with only $5 \mathrm{~mol} \%$ of FL17.

Here, the obvious long-range correlation between submicrometer domains of self-assembled fluorinated lipids can be treated as an analog of $2 \mathrm{D}$ colloidal crystallization of likecharged colloidal particles. ${ }^{13,14}$ In order to quantitatively evaluate the strength of interdomain correlations, the radial distribution function $g(r)$ from $>2000$ domains [Fig. 4(a)] is calculated $^{15}: g(r)=[d N(r+d r) / d(A+d A)] \times A / N$.

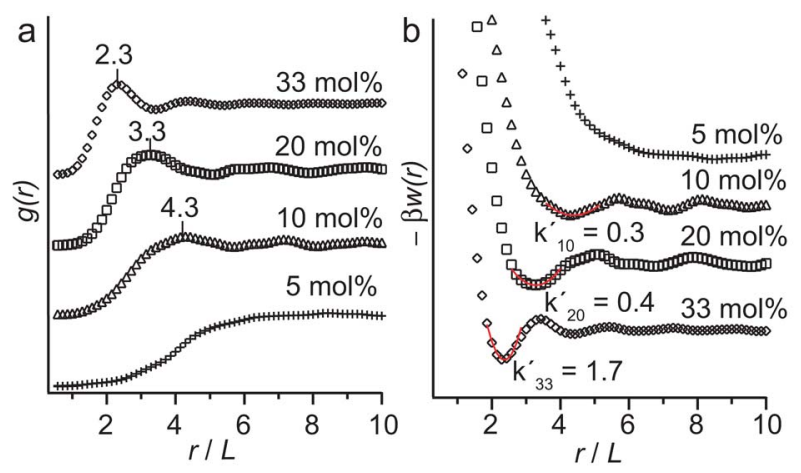

FIG. 4. (Color online) (a) The radial distribution function $g(r)$ of microdomains vs $r / L$. Note that a broad peak observed in Fig. 3 cannot be identified, as $g(r)$ takes all correlations into account. (b) The potential of mean force $-\beta w(r)$ vs $r / L$ calculated from the inverse work theorem $-\beta w(r)$ $=\ln [g(r)]$. More than one order can be observed at FL17 $\geq 10 \mathrm{~mol} \%$, indicating a strong correlation between self-assembled FL17 domains, ranging up to $\sim 8 r / L$. Spring constants $k^{\prime}$ derived from a harmonic potential fit of the first order minima systematically increase with FL17. 
Note that "all" the pair correlations are now taken into consideration, which is in contrast to $p(r)$ that merely counts correlations between the nearest neighbors. In fact, the broad peak at FL17 $=5$ mol \% seen in Fig. 3 cannot be resolved any longer in this plot, although the first order peaks are found almost at the identical positions. On the other hand, $g(r)$ enables us to identify correlations reaching out over much longer distances. Taking the inverse work theorem, $g(r)$ can be related to so-called potential of mean force $-\beta w(r):-\beta w(r)=\ln [g(r)]$, where $\beta=1 / k_{B} T$. As obvious from the definition, this potential is of free energy nature and includes many body interactions, namely, $w(r)$ equals to the direct pair potentials between two particles only for infinitely diluted system. ${ }^{16}$ As shown in Fig. 4(b), the position and width of the first order minimum increase with the decrease in FL17, while both the first and second order potential minima, indicating the onset of an ordering process, can be seen at FL17 $\geq 10 \mathrm{~mol} \%$. The potentials near the first order minima are well described as harmonic, ${ }^{17}$ whose spring constants $k^{\prime}$ systematically increase with FL17. It should be noted that the long-range correlation can reach up to the distance of $\sim 8 r / L$. On the other hand, as suggested by Fig. 4(a), uncorrelated effective interactions that follow Yukawa potential $^{18}$ can be observed only at FL17=5 mol \% [supporting information S2 (Ref. 7)].

Such strongly correlated domains of well defined size at various area densities can only be designed by taking advantage of the oleophobic nature of fluorinated lipids. Our preliminary experiments with other fluorinated lipids actually reveal that the size of domains can flexibly be tuned by the length of fluorocarbon chains up to a factor of 10 , which suggests the influence of the molecular length on the free energy of the line tension (Oelke et al., unpublished results). Further coupling of biofunctional moieties (e.g., saccharides and peptides) to the preformed domains will be straightforward to create biofunctional nano- and microdomains that can be utilized as a well defined model system to study the influence of molecular confinement on the cooperative biological functions, such as cell adhesion.

Synthesis of the fluorinated FL17 lipid followed the synthetic pathway reported by Huang et al. ${ }^{19}$ Brief descriptions can be found as the supporting information S3. ${ }^{7}$ 1,2-dioleoyl-sn-phosphatidylcholine (from Avanti Polar Lipids) was used as the fluid phospholipids matrix, and Texas red 1,2-dihexadecanoyl-sn-glycero-3-phosphoethanolamine, triethylammonium salt (TR-DHPE) was purchased from Invitrogen. Prior to the membrane deposition, cleaned glass slides were hydrophobized with octadecyltrimethoxysilane (ABCR, Karlsruhe, Germany) monolayer ${ }^{20}$ and a lipid mixture in $1: 1$ (v/v) 1,1,2-trichlorotrifluoroethane (Riedel-de Haën, Seelze, Germany): chloroform was deposited onto the water subphase of a Langmuir film balance (KSV Instruments, Helsinki, Finland). After evaporation of solvents, the film was compressed to $\pi=25 \mathrm{mN} / \mathrm{m}$ at $T=293 \mathrm{~K}$ at very low speed $\left(1 \AA^{2}\right.$ molecule $\left.^{-1} \mathrm{~s}^{-1}\right)$ in order to ensure that the system is close to the thermodynamic equilibrium. In fact, the pressure-area isotherms (Langmuir isotherms) and the size/ distribution of the domains were reproducible upon several compression/relaxation cycles. The film was transferred onto a hydrophobized glass slide by the horizontal dipping of the substrate.

GID measurements were carried out at ID $10 \mathrm{~B}$ at the European Synchrotron Research Facility (ESRF) in Grenoble, France. ${ }^{21}$ A monochromatic synchrotron x-ray beam $(\lambda=1.5 \AA)$ impinges to the monolayer at a grazing angle $\left(\alpha_{i}\right)$ slightly below the critical angle $\left(\alpha_{c}\right)$ of total reflection $\left(\alpha_{i}=0.8 \times \alpha_{c}\right)$, and the intensity of the diffracted beam is counted by a position sensitive detector oriented perpendicularly to the sample surface.

We thank ESRF for the beamtime, R.R. Schmidt for the insightful comments on lipid synthesis, and E. Schneck for supporting the GID data analysis. This work was financially supported by the German Science Foundation (DFG) Contract No. Ta253/6 and the Fonds der Chemischen Industrie. J.O. and A.W. gratefully acknowledge generous financial support of the German Excellence Initiative via the "Nanosystems Initiative Munich (NIM).”

${ }^{1}$ K. Simons and E. Ikonen, Nature (London) 387, 569 (1997).

${ }^{2}$ M. Mammen, S.-K. Choi, and G. M. Whitesides, Angew. Chem., Int. Ed. 37, 2754 (1998).

${ }^{3}$ C. Dietrich, L. A. Bagatolli, Z. N. Volovyk, N. L. Thompson, M. Levi, K. Jacobson, and E. Gratton, Biophys. J. 80, 1417 (2001).

${ }^{4}$ T. Baumgart, S. T. Hess, and W. W. Webb, Nature (London) 425, 821 (2003).

${ }^{5}$ S. L. Veatch and S. L. Keller, Phys. Rev. Lett. 89, 268101 (2002).

${ }^{6}$ L. K. Tamm and H. M. McConnell, Biophys. J. 47, 105 (1985).

${ }^{7}$ See EPAPS Document No. E-APPLAB-93-069846 for GID data (S1), comparison to Yukawa potential (S2), and synthesis of FL17 (S3). For more information on EPAPS, see http://www.aip.org/pubservs/epaps.html.

${ }^{8}$ C. W. Bunn and E. R. Howells, Nature (London) 174, 549 (1954).

${ }^{9}$ P. Fontaine, M. Goldmann, P. Muller, M. C. Faure, O. Konovalov, and M. P. Krafft, J. Am. Chem. Soc. 127, 512 (2005).

${ }^{10} \mathrm{~A}$. Guinier, Crystals, Imperfect Crystals, and Amorphous Bodies (Freeman, San Francisco, 1963).

${ }^{11}$ C. Zakri, A. Renault, J. P. Rieu, M. Vallade, B. Berge, J. F. Legrand, G. Vignault, and G. Grubel, Phys. Rev. B 55, 14163 (1997).

${ }^{12}$ P. Pieranski, Phys. Rev. Lett. 45, 569 (1980).

${ }^{13}$ L. Ramos, T. C. Lubensky, N. Dan, P. Nelson, and D. A. Weitz, Science 286, 2325 (1999).

${ }^{14}$ M. Quesada-Pérez, J. Callejas-Fernández, and R. Hidalgo-Álvarez, Adv. Colloid Interface Sci. 95, 295 (2002).

${ }^{15}$ M. Quesada-Pérez, A. Moncho-Jordá, F. Martínez-López, and R. HidalgoÁlvarez, J. Chem. Phys. 115, 10897 (2001).

${ }^{16}$ L. Belloni, J. Phys.: Condens. Matter 12, R549 (2000).

${ }^{17}$ M. G. Nikolaides, A. R. Bausch, M. F. Hsu, A. D. Dinsmore, M. P. Brenner, C. Gay, and D. A. Weitz, Nature (London) 420, 299 (2002).

${ }^{18}$ E. J. W. Verwey and J. T. G. Overbeck, Theory of the Stability of Lyophobic Colloids (Elsevier, Amsterdam, 1948).

${ }^{19}$ W. J. Huang, C. Y. Jin, D. K. Derzon, T. A. Huber, J. A. Last, P. P. Provencio, A. S. Gopalan, M. Dugger, and D. Y. Sasaki, J. Colloid Interface Sci. 272, 457 (2004).

${ }^{20}$ H. Hillebrandt and M. Tanaka, J. Phys. Chem. B 105, 4270 (2001).

${ }^{21}$ D. M. Smilgies, N. Boudet, B. Struth, and O. Konovalov, J. Synchrotron Radiat. 12, 329 (2005). 